\title{
B Cell-Activating Factor (BAFF) in Inflammatory Bowel Disease: BAFFling No Longer?
}

\author{
Ilja Striz
}

Published online: 24 June 2016

(c) Springer Science+Business Media New York 2016

Inflammatory bowel disease (IBD), manifest as Crohn's disease (CD) or ulcerative colitis (UC) results from multiple interactions between genetic and environmental factors such as the microbiome, and also from dysregulation of the immune system, inflaming the gut mucosa. Although cytokine imbalance, with a predominance of Th1, Th2, or Th17 pathways is associated with the overproduction of proinflammatory cytokines in the intestinal mucosa, the presence of antineutrophil cytoplasmic antibodies (ANCA) and anti-Saccharomyces cerevisiae (ASCA) antibodies implicate B cell responses as well. Furthermore, IBD is also associated with the dysfunction of gut regulatory immune cells, including intestinal intraepithelial lymphocytes, Foxp3(+) regulatory $\mathrm{T}$ cells, regulatory $\mathrm{B}$ cells, alternatively activated macrophages, dendritic cells, and innate lymphoid cells [1].

The clinical course of CD and UC is related not only to the topography of the affected intestine but also to the great variability of disease activity. In this respect, non-invasive biomarkers for the early detection and monitoring of IBD are still needed. The currently used routine biomarkers to assess IBD activity such as faecal calprotectin, C-reactive protein, and erythrocyte sedimentation rate, although useful as nonspecific inflammatory indicators, lack the specificity needed to predict relapse or to assess disease progression. Although the emergence of other biomarkers such as the autoantibodies and proinflammatory cytokines, lactoferrin, S100A12, granulocyte-macrophage colony-

Ilja Striz

ilja.striz@ikem.cz

1 Department of Clinical and Transplant Immunology, Institute for Clinical and Experimental Medicine, Videnska 1958/9, Prague, Czech Republic stimulating factor (GM-CSF), soluble CD14 (sCD14), and lipopolysaccharide-binding protein (LBP) have contributed to our understanding of IBD pathogenesis, none are of sufficient reproducibility to warrant clinical development as predictive biomarkers [2].

In this issue of Digestive Diseases and Sciences, Zhang and colleagues report the first study focused on B cellactivating factor (BAFF) as a new potential marker for monitoring of disease activity in IBD [3]. BAFF, produced primarily by innate immune cells, is a key cytokine governing adaptive responses by regulating mature B cell survival and differentiation into antibody-producing plasma cells. Since overproduction of BAFF in autoimmune diseases promotes the survival of self-reactive $B$ cells, targeting of BAFF by therapeutic monoclonal antibodies is currently being clinically tested [4]. Moreover, elevated BAFF concentrations are present in other chronic inflammatory conditions only vaguely related to B cells, such as chronic obstructive pulmonary disease [5].

The authors reported [3] that BAFF concentrations in serum and particularly in faeces discriminated active IBD patients from healthy controls and subjects with irritable bowel syndrome (IBS) with excellent specificity and sensitivity. In patients with UC, serum, but not faecal BAFF concentrations correlated with clinical disease activity and serum concentrations of C-reactive protein (CRP), tumour necrosis factor alpha (TNF- $\alpha$ ) and interleukin 1 beta (IL-1 $\beta$ ). Although the article provided data to support BAFF as a promising biomarker in IBD patients, it raised also two important questions: how does BAFF contribute to IBD immunopathogenesis and is BAFF as a potential therapeutic target?

The contribution of BAFF to IBD pathogenesis is supported by data obtained from colonic biopsies from $\mathrm{CD}$ and UC patients in which high mRNA and BAFF protein 
expression are present. In inflamed regions of UC mucosa, upregulation of BAFF was predominant in lamina propria mononuclear cells [3]. In this respect, although BAFF is widely accepted as a cytokine affecting principally $\mathrm{B}$ and $\mathrm{T}$ cells, BAFF also stimulates innate immune cells, documented by improvements of human monocyte survival, proinflammatory cytokine secretion, and upregulation of costimulatory molecules [6]. Monocyte-derived gastrointestinal macrophages and dendritic cells have important interactions with the microbial environment, induction of adaptive immune responses, proinflammatory tissue injury, and resolution of mucosal inflammation [7]. Although the presence of BAFF is preferentially associated with the induction of autoimmune inflammation, an alternative function includes induction of B regulatory cells protecting the intestinal mucosa from inflammatory injury. Recently, reduced frequencies of $\mathrm{B}$ regulatory cells in peripheral blood and the intestinal mucosa were documented in UC patients, accompanied by downregulation of IL-10 serum concentrations [4]. Contrariwise, the correlation of BAFF serum concentrations with clinical disease activity and standard laboratory disease biomarkers indicate that BAFF is primarily proinflammatory in IBD (Fig. 1).
In the last two decades, knowledge of the immunopathogenic mechanisms underlying inflammatory processes in IBD has greatly expanded, providing the basis for new targeted therapeutics that have been introduced into clinical practice. Nevertheless, most of the biologics are designed to inhibit either proinflammatory cytokines or affect the regulation of Th1/Th17 pathways. Although the human monoclonal BAFF antibody belimumab was approved for systemic lupus erythematosus, having a low incidence of organ damage and an excellent safety profile [8], other BAFF inhibitors were much less beneficial in clinical trials, suggesting redundancies in B cell signalling/survival pathways, which should be better understood before future clinical trials are conducted [9]. Recently, blisibimod, a potent and selective BAFF inhibitor with a unique tetravalent, "peptibody" structure, has been introduced in clinical evaluation for the treatment of systemic lupus erythematosus (SLE) [10].

The main contribution of the manuscript to the field of IBD is the description of a new specific and sensitive clinical biomarker that has not been previously tested. Moreover, the local overproduction of BAFF in the mucosa of patients with $\mathrm{CD}$ and UC together with elevated serum

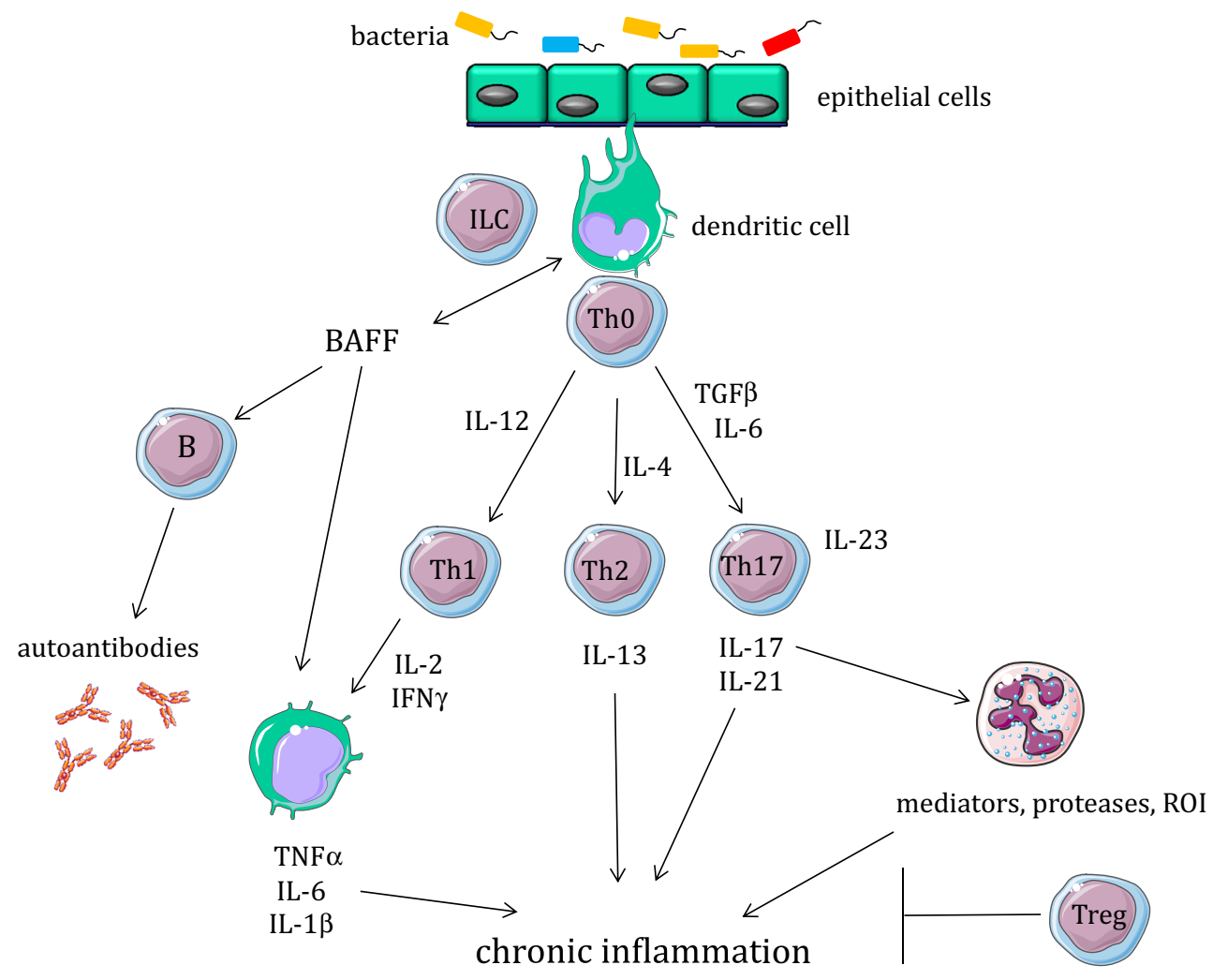

Fig. 1 The potential involvement of BAFF in the immunopathogenesis of inflammatory bowel disease 
concentrations of BAFF also provides a basis for the development of BAFF-targeted therapies for IBD.

\section{References}

1. Sun M, He C, Cong Y, Liu Z. Regulatory immune cells in regulation of intestinal inflammatory response to microbiota. $\mathrm{Mu}$ cosal Immunol. 2015;8:969-978.

2. Musci JO, Cornish JS, Dabritz J. Utility of surrogate markers for the prediction of relapses in inflammatory bowel diseases. $J$ Gastroenterol. 2016;51:531-547.

3. Zhang P, Liu X, Guo A, Xiong J, Fu Y, Zou K. B cell-activating factor as a new potential marker in inflammatory bowel disease. Dig Dis Sci. (Epub ahead of print). doi:10.1007/s10620-0164136-z.

4. Liu Z, Davidson A. BAFF and selection of autoreactive B cells. Trends Immunol. 2011;32:388-394.
5. Polverino F, Cosio BG, Pons J, et al. B cell-activating factor. An orchestrator of lymphoid follicles in severe chronic obstructive pulmonary disease. Am $J$ Respir Crit Care Med. 2015;192:695-705.

6. Chang SK, Arendt BK, Darce JR, Wu X, Jelinek DF. A role for BLyS in the activation of innate immune cells. Blood. 2006;108:2687-2694.

7. Steinbach EC, Plevy SE. The role of macrophages and dendritic cells in the initiation of inflammation in IBD. Inflamm Bowel Dis. 2014;20:166-175.

8. Barranco C. Connective tissue diseases: belimumab might retard SLE damage accrual. Nat Rev Rheumatol. 2016;12:194.

9. Singh N, Kumar B, Aluri V, Lenert P. Interfering with baffled B cells at the lupus tollway: promises, successes, and failed expectations. J Allergy Clin Immunol. 2016;137:1325-1333.

10. Scheinberg MA, Hislop CM, Martin RS. Blisibimod for treatment of systemic lupus erythematosus: with trials you become wiser. Expert Opin Biol Ther. 2016;16:723-733. 